\title{
Noninvasive imaging technique can identify high-risk coronary plaques
}

Atherosclerotic plaque rupture and subsequent acute myocardial infarction are well known to be a major cause of death worldwide. Prediction of plaque rupture, however, has proven challenging. A new noninvasive imaging technique that uses an inexpensive PET radioisotope, ${ }^{18} \mathrm{~F}-\mathrm{NaF}$, might prove to be the answer. Indeed, the investigators of a study published in the Lancet believe that their technique "holds major promise as a means of identifying highrisk and ruptured plaque, and potentially informing the future management and treatment of patients with stable and unstable coronary artery disease".

When 40 patients who had been hospitalized for myocardial

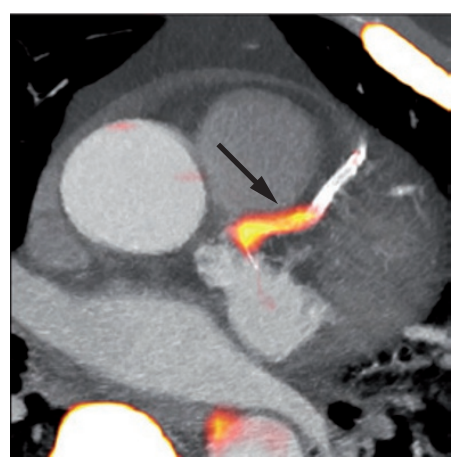

Image reprinted from Joshi, N. V. et al. ${ }^{18} \mathrm{~F}$-fluoride positron emission tomography for identification of ruptured and high-risk coronary atherosclerotic plaques: a prospective clinical trial. Lancet doi:10.1016/S0140-6736(13)61754-7,

Copyright (2013), with permission from Elsevier. infarction underwent ${ }^{18} \mathrm{~F}-\mathrm{FDG}$ PET-CT scanning, no significant differences in maximum ${ }^{18} \mathrm{~F}$-FDG uptake were observed between the culprit plaques and plaques located elsewhere in the coronary vasculature. By contrast, increased ${ }^{18} \mathrm{~F}-\mathrm{NaF}$ uptake was observed in the culprit plaque in 37 of the 40 patients. ${ }^{18} \mathrm{~F}-\mathrm{NaF}$ activity was found to be $34 \%$ higher in the culprit plaque than the maximum activity recorded anywhere else in the coronary vasculature.

Ex vivo ${ }^{18} \mathrm{~F}-\mathrm{NaF}$ PET-CT and histological analysis were performed on carotid specimens obtained from nine other patients who had undergone carotid endarterectomy for symptomatic carotid artery disease. Compared with tissue that did not take up ${ }^{18} \mathrm{~F}-\mathrm{NaF}$, areas of increased ${ }^{18} \mathrm{~F}-\mathrm{NaF}$ uptake were found to have increased calcification, macrophage infiltration, and cell death. In their study report, Dr Nikhil Joshi and colleagues describe how they believe ${ }^{18} \mathrm{~F}-\mathrm{NaF}$ is taken up by high-risk plaques: "Hydroxyapatite is the central structural component of vascular calcification and is laid down during the earliest and most active stages of mineralization ... Fluoride ions are incorporated into the hydroxyapatite by ion exchange with hydroxyl groups at the crystal surface." "By identifying areas of nascent and ongoing calcification activity," they explain, " ${ }^{18} \mathrm{~F}-\mathrm{NaF}$ uptake allows us to detect regions of metabolically active plaque."

Joshi et al. also assessed coronary ${ }^{18} \mathrm{~F}-\mathrm{NaF}$ uptake in 40 patients with stable angina pectoris. Increased ${ }^{18} \mathrm{~F}-\mathrm{NaF}$ uptake seemed to identify coronary plaques that, although mostly shown to be nonobstructive on coronary angiography, contained high-risk features (increased positive remodelling, microcalcification, and necrotic core) on intravascular imaging.

"Prospective studies are now needed in a broad range of patients to assess whether increased coronary ${ }^{18} \mathrm{~F}-\mathrm{NaF}$ activity will ultimately translate into future adverse events," say the investigators. "If the results prove confirmatory, then this technique has the potential to fundamentally alter the way we treat coronary artery disease."

Bryony M. Mearns

Original article Joshi, N. V. et al. ${ }^{18} \mathrm{~F}$-fluoride positron emission tomography for identification of ruptured and high-risk coronary atherosclerotic plaques: a prospective clinical trial. Lancet doi:10.1016/S01406736(13)61754-7 